\title{
Erythrocyte Duffy antigen receptor for chemokines (DARC): diagnostic and therapeutic implications in atherosclerotic cardiovascular disease
}

\author{
Stavros APOSTOLAKIS, Georgios K CHALIKIAS, Dimitrios N TZIAKAS*, Stavros KONSTANTINIDES \\ Department of Cardiology, Medical School, Democritus University of Thrace, Alexandroupolis, Greece
}

Atherosclerosis is an inflammatory disease. The last three decades efforts have been made to elucidate the biochemical pathways that are implicated in the process of atherogenesis and plaque development. Chemokines are crucial mediators in every step of this process. Additionally, cellular components of the peripheral blood have been proved important mediators in the formation and progression of atherosclerotic lesions. However, until recently data were mostly focusing on leukocytes and platelets. Erythrocytes were considered unreceptive bystanders and limited data supported their importance in the progression and destabilization of the atherosclerotic plaque. Recently erythrocytes, through their Duffy antigen receptor for chemokines (DARC), have been proposed as appealing regulators of chemokine-induced pathways. Dissimilar to every other chemokine receptor DARC possesses high affinity for several ligands from both CC and CXC chemokine sub-families. Moreover, DARC is not coupled to a G-protein or any other intracellular signalling system; thus it is incapable of generating second messages. The exact biochemical role of erythrocyte DARC remains to be determined. It is however challenging the fact that DARC is a regulator of almost every CC and CXC chemokine ligand and therefore DARC antagonism could effectively block the complex pre-inflammatory chemokine network. In the present review we intent to provide recent evidence supporting the role of erythrocytes in atherosclerosis focusing on the erythrocyte-chemokine interaction through the Duffy antigen system.

Keywords: erythrocytes; chemokines; Duffy antigen receptors for chemokines; atherosclerosis

Acta Pharmacologica Sinica (2011) 32: 417-424; doi: 10.1038/aps.2011.13; published online 28 Mar 2011

\section{Introduction}

The Duffy blood group system was first reported by Cutbush and Mollison in $1950^{[1]}$. The authors reported an alloantibody against an antigen denoted as $\mathrm{Fy}^{\mathrm{a}}$ in a multiple transfused patient with haemophilia. The antigen was named after the patient. The antithetical antigen, $\mathrm{Fy}^{\mathrm{b}}$, was described one year later. Three phenotypes were identified in Caucasians: $\mathrm{Fy}^{\mathrm{a}}{ }^{\mathrm{a}-}$, $\mathrm{Fy}^{\mathrm{a}-\mathrm{b}+}$, and $\mathrm{Fy}^{\mathrm{a}+\mathrm{b}+}$. These phenotypes are the products of codominant alleles comprising genotypes $\mathrm{FY}^{*} \mathrm{~A} / \mathrm{FY}^{*} \mathrm{~A}, \mathrm{FY}{ }^{*} \mathrm{~B} /$ $\mathrm{FY}^{*} \mathrm{~B}$, and $\mathrm{FY}^{*} \mathrm{~A} / \mathrm{FY}{ }^{*} \mathrm{~B}$, respectively. The $\mathrm{Fy}^{\mathrm{a}-\mathrm{b}-}$ phenotype, is extremely rare in Caucasians and occurs primarily as a result of GATA promoter region mutation upstream of the FY allele. This mutation prevents expression of Duffy glycoprotein on erythrocytes only, while permitting expression of nonerythroid cells. Most West Africans and 68\% of African Americans do not express Duffy antigens on their erythrocytes ${ }^{[2,3]}$. Interestingly recent data suggest that reduced neutrophil count in

\footnotetext{
* To whom correspondence should be addressed.

E-mail dtziakas@med.duth.gr

Received 2010-11-28 Accepted 2011-02-14
}

people of African descent is due to a regulatory variant in the Duffy antigen receptor gene ${ }^{[4]}$.

Soon, the Duffy blood group antigen was identified as a potent multi-ligand chemokine receptor and was therefore renamed to Duffy antigen receptor for chemokines (DARC) ${ }^{[5]}$.

DARC belongs to the family of rhodopsin-like seven-helix transmembrane proteins ${ }^{[5,6]}$. Besides erythrocytes DARC is expressed in postcapillary venular endothelial cells, which are the primary site of leukocyte transmigration in most tissues. The Duffy antigen has emerged as a highly specific binding site for both CC and CXC chemokines. The Duffy antigen possesses higher affinity for ELR motif CXC chemokines, which are neutrophil chemoattractants and presumably proangiogenic. The best studied interaction between DARC and CXCL8 has demonstrated a dissociation constant $\left(K_{\mathrm{d}}\right)$ of 5 $\mathrm{nmol} / \mathrm{L}$ and receptor binding sites estimated at 1000-9000 per erythrocyte $^{[7]}$. Unlike all other seven-transmembrane chemokine receptors, DARC lacks the highly conserved G proteincoupling motif located in the second cytoplasmic loop ${ }^{[6-8]}$. Thus DARC is not G-protein coupled and has no known alternative signalling mechanism. The biological role of a 
chemokine receptor that is unable to generate a second message and promote chemotaxis became a challenging field of investigation $^{[6-8]}$.

In the present review we intent to provide recent evidence supporting the role of erythrocytes in atherosclerosis focusing on the erythrocyte-chemokine interaction through the Duffy antigen system; a novel and challenging regulatory mechanism with potential implications in the management of atherosclerotic cardiovascular disease.

\section{Erythrocytes and atherosclerosis}

\section{Erythrocytes: new players in the atherosclerotic cascade}

Despite that knowledge on the pathophysiological background of atherosclerosis is exponentially growing, several aspects of the natural history of the disease remain unclear ${ }^{[9]}$. Cellular components of the peripheral blood have been proved important mediators in the genesis and progression of atherosclerotic lesions. However, until recently data were mostly focusing on leukocytes and platelets. Erythrocytes were considered unreceptive bystanders and limited data supported their importance in the progression and destabilization of the atherosclerotic plaque ${ }^{[10]}$.

Roberts and Virmani were the first to report the presence of extravasated erythrocytes and iron within coronary atherosclerotic plaques ${ }^{[11]}$. The authors further demonstrated that the amount of intraplaque extravasated erythrocytes and iron was proportional to the extent of coronary atherosclerotic plaque ${ }^{[11]}$. Accordingly, Arbustini et al, identified erythrocyte membranes in atherosclerotic plaques outside the coronary circulation and suggested that cholesterol contained in their membranes may contribute significantly to the formation of the atheromatous core $^{[12]}$. Kolodgie et al, reported that erythrocyte membranes were present in the necrotic core of advanced coronary atheromas and that their presence was correlated with the size of the necrotic core ${ }^{[13]}$. Subsequently a theory was raised suggesting that erythrocyte membranes contributed significantly to the growth of the plaque's core and subsequently contribute to its vulnerability ${ }^{[14]}$.

\section{Erythrocytes: mediators of plaque vulnerability}

The erythrocyte membrane is a simple plasma membrane comprised mainly of cholesterol and phospholipids ${ }^{[15]}$. The erythrocyte membranes are 1.5-2.0 times richer in cholesterol than any other membrane in the body and approximately $40 \%$ of its weight is composed of lipids ${ }^{[16]}$. The volume of cholesterol in the membrane of a single erythrocyte is estimated to be $0.378 \mu \mathrm{m}^{3}$ whilst the majority of its cholesterol is considered to be free ${ }^{[16]}$. Only $50 \mu \mathrm{L}$ erythrocytes are needed to produce a $\geq 0.2 \mathrm{~mm}^{3}$ necrotic core ${ }^{[17]}$. Therefore, breakdown of the erythrocytes within advanced atheromatous lesions can result in substantial cholesterol accumulation. We have previously demonstrated that-in the clinical setting-cholesterol content of erythrocyte membranes is higher in patients with acute coronary syndromes compared to those with stable coronary artery disease ${ }^{[18,19]}$. At the same time, degradation of erythrocytes inside the atheromas may lead to haemoglobin and iron release which is known to promote inflammation, further oxidation of lipids, free radicals production and activation of apoptotic pathways ${ }^{[13,20]}$.

\section{Erythrocytes: a sink for pro-inflammatory molecules}

Erythrocytes can also regulate inflammation within the plaque. Through the Duffy antigen receptor for chemokines (DARC) erythrocytes can bind and curry without deactivating a wide range of CXC and CC chemokines ${ }^{[8,21]}$. The accumulation of erythrocytes within the plaques is the natural consequence of intraplaque haemorrhage. Thus it is reasonable to hypothesize that erythrocytes entering the necrotic lipid core can release within the plaque a substantial amount of their pro-inflammatory load. Therefore, erythrocyte emerges as a non-inflammatory cellular regulator of the local inflammatory processes acting as a bidirectional sink for chemokines ${ }^{[22]}$.

Moreover erythrocytes are a major source of microparticles ${ }^{[23]}$. Microparticles or microvesicles are circulating, phospholipid rich, submicron elements released from the membranes of endothelial cells, platelets, leucocytes and erythrocytes ${ }^{[23]}$. Microvesicles play a role in intercellular communication and can deliver mRNA, miRNA, and proteins between cells ${ }^{[23]}$. These actions are mediated through their phospholipid rich surfaces and the expression of cell surface molecules which reflect their cell of origin and its state of activation. Investigation into their biological activity has also revealed diverse actions in coagulation ${ }^{[23]}$. Their procoagulant properties rely on the exposure of phosphatidylserine and on the possible presence of tissue factor, the main initiator of blood coagulation. Microparticles constitute the main reservoir of blood-borne tissue factor ${ }^{[23]}$. Elevated levels of microparticles have been encountered in systemic inflammatory disease, especially diseases with vascular involvement including acute coronary syndromes, where they appear indicative of a poor clinical outcome ${ }^{[23-26]}$. In the context of atherothrombosis both intact erythrocytes and erythrocyte derived microparticles could be acted as vehicles transferring DARC-bound chemokine ligands in sites of vascular damage. The chemokine content of erythrocyte microvesicles in vivo has not been assessed yet. Nevertheless, erythrocyte microparticles express $\mathrm{DARK}^{[24]}$. Moreover, in vitro and ex vivo data suggest that erythrocyte microvesicles have altered membrane properties compared to intact erythrocyte membranes and under stimulation they can increase chemokine bioavailability ${ }^{[25]}$.

Atherosclerosis can also affect the morphology and function of erythrocytes. It has been proposed that reactive oxygen species produced in the course of atherosclerosis, lead to excessive cytoskeletal protein modification of erythrocytes. The modified erythrocytes are abnormally prone to vesiculation during mechanical stress in vitro and apparently in vivo. Although these data were originally obtained from sickle cell anaemia models this abnormality was successfully reproduced in normal erythrocytes by causing stress conditions using phenazine methosulfate (PMS)-induced stimulation of intracellular superoxide generation, a process similar to that occurring in sickle erythrocytes. Thus it could be that the genera- 
tion of reactive oxygen species in atherosclerosis activates red blood cells, and microvesicles of red blood cells are formed, enhancing the activation of the vascular endothelium and leading to vascular inflammation and atherogenesis ${ }^{[26]}$.

\section{CXC chemokines and their receptors in atherosclerosis Chemokine network in atherosclerosis}

Chemokines are small secreted chemoattractant proteins (approximately 8-17 kDa) that serve as regulatory molecules in cellular trafficking and activation. Chemokines share considerable homology and most importantly, a conserved tetracysteine motif. They are classified into four sub-families based on the number and structural arrangement of conserved cysteine residues within their amino-terminal polypeptide sequence (Table 1$)^{[27-29]}$. Chemokines induce cell activation by binding to specific seven-transmembrane G-protein coupled cell-surface receptors on target cells. Chemokines interact with their receptors on the cell surface leading to the generation of an intracellular signal via the G-protein complex, and subsequently to cell chemotaxis towards a chemokine gradient ${ }^{[27-29]}$. Cell movement and migration is driven by the dynamic assemblies of the actin cytoskeleton. In chemotaxis, chemokine gradients strongly bias this actin assembly to the cell's leading edge and, hence modify the direction of cell movement ${ }^{[30]}$. Decoy receptors-also known as interceptors (internalizing receptors)-, which bind chemokine ligands with high affinity but do not elicit signal transduction, include D6, DARC, and CCX-CKR ${ }^{[31]}$. Chemokine receptor CCX-CKR is a scavenger of CCR7 ligand chemokines while D6 is thought to act as a chemokine scavenger for pro-inflammatory CC chemokines. DARC is considered a decoy receptor for both CC and CXC motif chemokines while no data exist on its affinity for CX3C and XC chemokines (Table 1).

In the context of atherosclerotic cardiovascular disease the role of chemokine signalling is complex and not fully elucidated. In fact it seems that each stage of atherosclerosis is characterized by different chemokine-signalling ${ }^{[32]}$. In the early stages of atherosclerosis, oxLDL induces the expression of CCL2 (or monocyte chemoattractant protein 1) and CX3CL1, by vascular smooth muscle cells (SMCs) and endothelial cells $(\mathrm{ECs})^{[32,33]}$. CX3CL1 is a structurally unique chemokine that acts both as a chemoattractant and as a potent adhesion molecule through a non-integrin-dependent mechanism ${ }^{[34-36]}$. CCL2, secreted by ECs and SMCs, promotes structural changes in the cytoskeleton of CCR2-positive monocytes, potentiating transendothelial migration ${ }^{[34-36]}$. The interaction of CCL5 (or regulated on activation normal T cell expressed and secreted), with its receptor CCR1 is also considered an early pathway leading to the firm adhesion of rolling monocytes to stimulated ECs. Concurrently, CXC chemokines induced by interferon gamma, such as CXCL9, CXCL10, and CXCL11 expressed predominantly by ECs interact with CXCR3-positive $\mathrm{T}$ cells, inducing their accumulation and migration, and subsequently increasing the vascular inflammatory response ${ }^{[34-36]}$. Recruitment of neutrophils and vascular progenitor cells in atherosclerotic lesions is controlled by
CXCR2 and CXCR4, and their ligands CXCL8 (or interleukin 8) and CXCL1 (or growth related oncogene-alpha). CXCL8 is highly expressed by lesion macrophages, as well as by ECs and SMCs. CXCL8 also promotes angiogenesis in advanced stages of plague development ${ }^{[33]}$. It is obvious that in the course of atherosclerosis, chemokines form a complicated network. Different chemokines promote different pathways. Moreover, the interaction of the same chemokine ligand with different receptors results in a different outcome. In such a complex system the consequences of a single pathway blockade cannot be predicted.

\section{CXC chemokines in atherosclerosis}

The CXC chemokines are the second largest class (Table 1$)^{[33]}$. Since CXC chemokines are mainly neutrophil chemoattractants, they have received less attention concerning their role in the pathogenesis of atherosclerosis. However accumulating evidence-focusing mostly on CXCL8-supports a crucial role of CXC chemokines in vascular pathology. In 1996, Rus et al first reported high levels of CXCL8 in the human arterial atherosclerotic wall, as cellular and extracellular deposit in the connective tissue matrix ${ }^{[37]}$. Similarly, Apostolopoulos et al demonstrated the expression of CXCL8 in human atherosclerotic plaques by in situ hybridization and also identified the macrophages as the main source of CXCL8 in atherosclerotic plaques ${ }^{[38]}$. CXCL8 has been further shown to promote firm adhesion of rolling monocytes to endothelial monolayers expressing E-selectin while it was proposed to contribute in SMC proliferation and migration in more advance stages of atherogenesis ${ }^{[39,40]}$. Boisvert et al provided direct evidence on the role of CXCL8 in atherosclerosis, demonstrating that LDL receptor knockout mice which were irradiated and repopulated with bone marrow cells lacking the murine homologue of CXCL8 receptor CXCR2 had less extensive lesions and fewer macrophages than those mice receiving bone marrow cells expressing the receptor ${ }^{[41]}$. Schwartz et al reported that CXCL1 an alternative ligand of CXCR2, contribute to the adhesion of monocytes to minimally modified-LDL stimulated ECs ${ }^{[42]}$. Similarly, Huo et al, using isolated carotid arteries from ApoE knockout mice demonstrated that CXCL1 but not CCL2 promotes monocyte arrest on the endothelium of atherosclerosis-prone vessels ${ }^{[43]}$. CXCL8 is not the only CXC chemokine that has been implicated in the pathways of atherogenesis. The chemokine receptor, CXCR3, has multiple highaffinity ligands including CXCL9 (or monokine induced by IFN-gamma), CXCL10 (or 10-kDa IFN-gamma-inducible protein) and CXCL11 (IFN-gamma-inducible T cell -chemoattracant). Mach et al demonstrated different levels of expression of CXCL9, CXCL10, and CXCL11 by atheroma-associated cells and suggested a potential role for these 3 interferon gammainducible CXC chemokines in the recruitment and retention of activated T lymphocytes in atherosclerotic lesions ${ }^{[44]}$. Platelets represent both a source and a target of CXC chemokines $^{[45]}$. Abi-Younes et al reported that, of the 16 chemokines tested, only CXCL12 (or stromal cell derived factor-alpha) induced platelet aggregation and proposed an involvement of 
Table 1. Chemokine ligands and receptors.

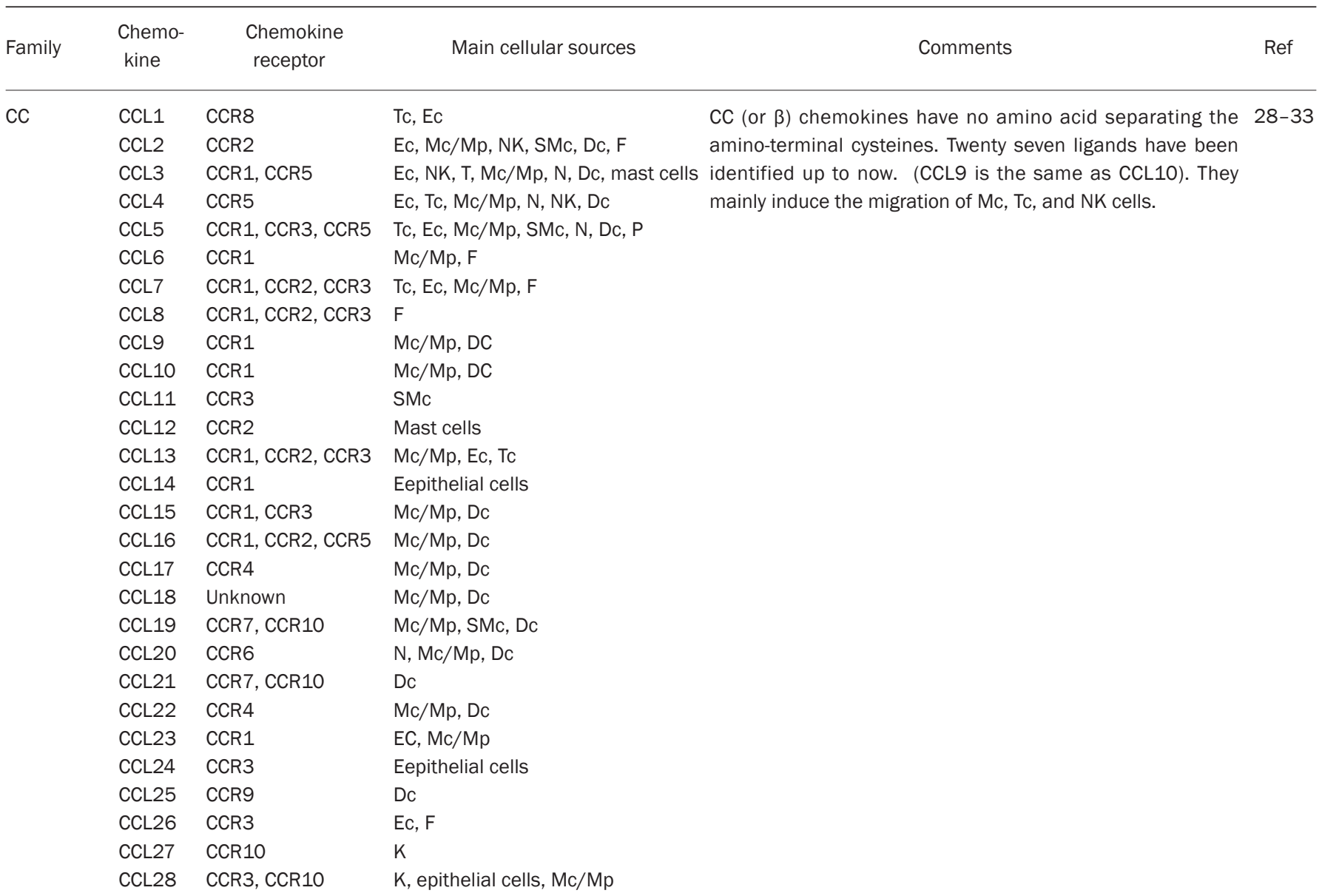

CXC

CXCL1 CXCR1

CXCL2 CXCR2

CXCL3 CXCR2

CXCL4 CXCR2

CXCL5 CXCR3

CXCL6 CXCR1, CXCR2

CXCL7 CXCR2

CXCL8 CXCR1, CXCR2

CXCL9 CXCR3

CXCL10 CXCR3

CXCL11 CXCR3, CXCR7

CXCL12 CXCR4, CXCR7

CXCL13 CXCR5

CXCL14 Unknown

CXCL15 Unknown

CXCL16 CXCR6

CXCL17 Unknown

C

XCL1 XCR1

XCL2 XCR1

$\mathrm{CX} 3 \mathrm{C}$
Ec, Mc/Mp, NK, F

Ec

$\mathrm{Mc} / \mathrm{Mp}$

$\mathrm{Tc}, \mathrm{P}, \mathrm{Mc} / \mathrm{Mp}$

$\mathrm{N}$

Ec

Megakaryocytes

Tc, Mc/Mp, Ec, Dc, N, SMc, F

$\mathrm{Ec}, \mathrm{Mc} / \mathrm{Mp}, \mathrm{N}, \mathrm{SMc}, \mathrm{F}$

$\mathrm{Tc}, \mathrm{Ec}, \mathrm{Mc} / \mathrm{Mp}, \mathrm{DC}$

$\mathrm{Ec}, \mathrm{Mc} / \mathrm{Mp}, \mathrm{SMc}$, mast cells, $\mathrm{F}$

$\mathrm{Ec}, \mathrm{SMc}, \mathrm{Mc} / \mathrm{Mp}, \mathrm{Dc}$,

$\mathrm{Mc} / \mathrm{Mp}, \mathrm{Dc}$

$\mathrm{F}$

Epithelial cells

$\mathrm{Mc} / \mathrm{Mp}, \mathrm{Dc}$

Dc

NK, Tc

NK, Tc

$\mathrm{Ec}, \mathrm{Mc} / \mathrm{Mp}, \mathrm{SMc}, \mathrm{Dc}$
CXC (or $\alpha$ ) chemokines have a single amino acid separating $28-33$ the two amino-terminal cysteine residues of the protein. They are further distinguished by the presence or absence of an amino acid sequence, glutamic acid-leucine arginine (ELR motif), which precedes the CXC sequence. ELR motif CXC chemokines are chemotactic in vitro for neutrophils but not for mononuclear cells. ELR negative CXC chemokines exhibit chemotactic activity toward monocytes and lymphocytes.
C chemokines lack two of the four conserved cysteines in the 28 mature protein. They recruit Tc and NK cells.

Include an amino-terminal domain with a novel arrangement 28,33 of three amino acids separating their first two cysteines. It exists as membrane-bound and in soluble form acting both as a chemoattractant and as an adhesion molecule mainly for $\mathrm{Mc} / \mathrm{Mp}$ and Tc. 
Table 1. Chemokine ligands and receptors.

\begin{tabular}{|c|c|c|c|c|c|}
\hline Family & $\begin{array}{l}\text { Chemo- } \\
\text { kine }\end{array}$ & $\begin{array}{l}\text { Chemokine } \\
\text { receptor }\end{array}$ & Main cellular sources & Comments & Ref \\
\hline \multirow{3}{*}{\multicolumn{2}{|c|}{ Interceptors }} & D6 & & A chemokine scavenger for pro-inflammatory CC chemokines. & 31 \\
\hline & & DARC & & Decoy receptor for both CC and CXC motif chemokines. & 21,22 \\
\hline & & CCX-CKR & & A scavenger of CCR7 ligand chemokines. & 31 \\
\hline
\end{tabular}

Tc, T lymphocytes; NK, natural killer cells; Ec, endothelial cells; N, neutrophils; Mc/Mp, monocytes/macrophages; P, platelets; F, fibroblasts; Dc, dendritic cells; K, keratinocyte; DARC, Duffy antigen receptor for chemokines.

CXCL12 in the pathogenesis of atherosclerosis and thromboocclusive disease ${ }^{[46]}$. Additionally, Schober et al assessed the role of CXCL12 in neointimal formation after vascular injury. CXCL12 was detected in the carotid arteries of ApoE knockout mice after wire-induced injury and was mostly located at sites of SMCs accumulation. Treatment of ApoE knockout mice after carotid injury with a neutralizing CXCL12 monoclonal antibody reduced neointimal lesion area, and decreased neointimal SMC content without influencing the relative content of neointimal macrophages. Thus, the authors concluded that CXCL12 played an active role in neointimal formation after vascular injury ${ }^{[4]}$. CXCL4 (or platelet factor 4) a CXCchemokine member secreted by platelets, induces monocyte activation, firm adherence of neutrophils on shear stressed ECs, and release of neutrophil granule components ${ }^{[48,49]}$. Pitsilos et al detected CXCL4 in the cytoplasm of luminal and neovascular endothelium, in macrophages and in regions of plaque calcification ${ }^{[50]}$. Yu et al demonstrated that E-selectin, is up-regulated in human umbilical vein endothelial cells exposed to CXCL4 ${ }^{[51]}$. Finally, Nassar et al showed that CXCL4 bound to oxidized LDL directly, and also increased oxidized LDL binding to vascular cells and macrophages, thus demonstrating an alternative mechanism by which platelet activation at sites of vascular injury may promote the accumulation of lipoproteins ${ }^{[52]}$.

\section{Erythrocytes as reservoirs/regulators of CXC chemokines DARC receptors on erythrocytes}

The current theory on erythrocytic DARC suggests that it acts as a regulator of chemokine activity. Since DARC is unable to initiate intracellular signals it is considered a negative regulator of chemokine signalling. However, current experimental data are unclear and sometimes conflicting and the exact role of erythrocyte DARC in vivo remains unclear. The function of this chemokine reservoir on the erythrocyte surface, and the fate of these chemokines at the end of the erythrocyte lifespan are essential pathways that still need to be elucidated. Increasing experimental evidence suggests that DARC on venular endothelium mediates chemokine internalization at the abluminal surface followed by transcytosis and transport of the chemokine load onto the luminal surface. DARC expressed on the erythrocyte membrane binds plasma chemokines resulting in neutralization of chemokines in the blood. This leads to leukocyte protection from unintended "desensitization" and enhancement of leukocyte recruitment ${ }^{[53]}$. The current concept on Duffy antigen's biological role is summarized in Figure 1.

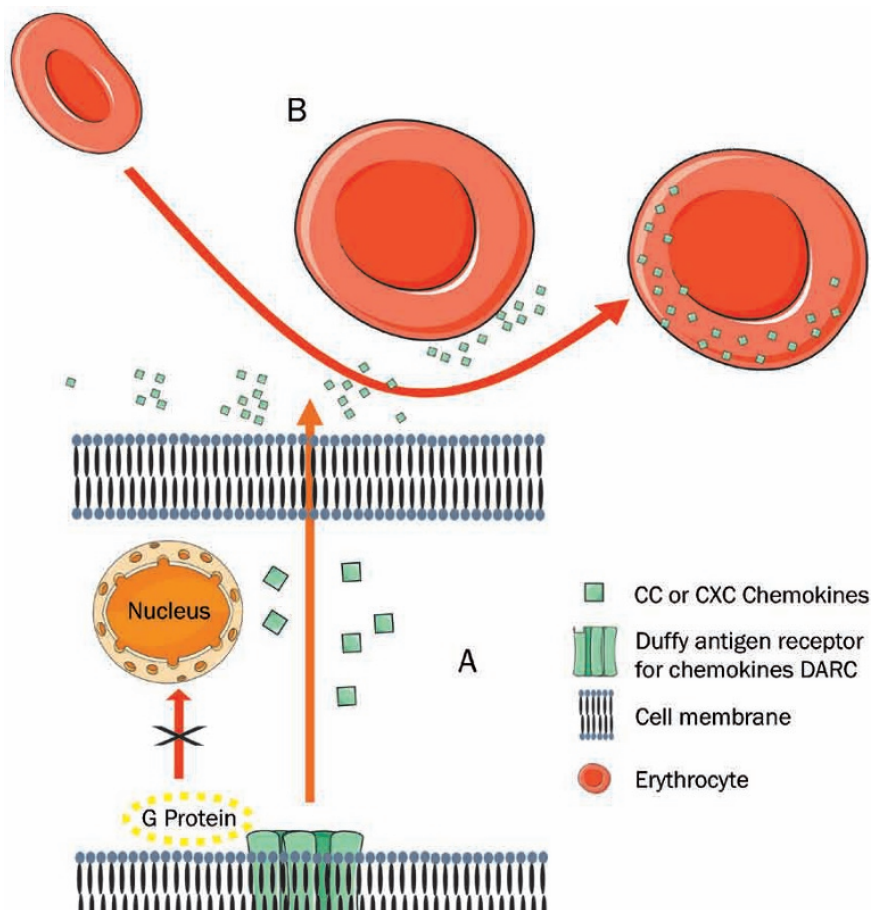

Figure 1. Schematic presentation of Duffy antigen's presumed biological action: Duffy antigen expressed on endothelial cells binds and internalizes chemokine ligands creating a transendothelial chemokine gradient (A), while Duffy antigen expressed on erythrocytes binds excess circulating chemokines. Despite structural homology with G-coupled chemokine receptors Duffy antigen cannot create a secondary message.

An important issue that had to be addressed early in DARC research was the impact of Duffy antigen activity on levels of circulating chemokines. If DARC positive erythrocytes serve as a reservoir for circulating chemokines then individuals carrying the DARC negative genotype or DARC loss of function polymorphisms should have higher levels of chemokines following an inflammatory stimulus. Dawson et al assessed indirectly this matter in a model of DARC negative mice. The authors demonstrated an increased number of neutrophils in organs of DARC deficient mice following intraperitoneal 
injection of lipopolysaccharide (LPS) ${ }^{[54]}$. Similarly in a model of human disease Nebor et al reported higher plasma levels of CXCL8 and CCL5 in Duffy negative patients with sickle cell anaemia ${ }^{[55]}$. Accordingly, in a genome wide analysis to identify the genetic basis of variance in circulating concentrations of CCL2, Schnabel et al concluded that the stronger predictor of CCL2 levels was a nonsynonymous polymorphism, (rs12075) in DARC gene ${ }^{[56]}$. Similarly, Lee et al utilized an in vitro model based on whole blood derived from humans currying "loss-of-function" genotypes of the DARC gene. The authors reported that plasma CXCL8 and CCL2 concentrations from humans homozygous for a "loss of function" DARC polymorphism were higher than in heterozygotes following LPS stimulation of their whole blood in vitro ${ }^{[57]}$. Finally, direct evidence of the impact of DARC phenotype on chemokine activity came from a pharmacokinetic study. Mayr et al assessed the impact of DARC phenotype on the pharmacokinetics of infused recombinant human CCL2. In a randomized, doubleblinded, placebo-controlled dose escalation trial the authors demonstrated that DARC negative individuals reached maximal plasma levels significantly earlier. Despite the fact that overall plasma concentration profiles were not altered the investigators provided direct evidence that DARC influences the kinetics of chemokines in vivo ${ }^{[58]}$. Moreover, the latter observation implies the presence of alternative pathways of chemokine homeostasis and disserves further investigation.

\section{DARC and atherosclerosis: In vitro and in vivo derived evidence}

Chemokine signalling has been a critical part of vascular inflammation and a leading field of investigation the last three decades. A regulatory point in the early stages of atherogenesis is accumulation and migration of circulating leukocytes into the intima of the vessel wall ${ }^{[33]}$. Chemokines promote this stage of atherogenesis by creating a chemotactic gradient that mobilizes the inflammatory cells towards the area of increased chemokine concentration ${ }^{[33]}$. Endothelial DARC has been proved essential in creating and preserving a transcellular chemokine gradient that promotes intercellular or transcellular leukocyte migration. Pruenster et al demonstrating that DARC, like other silent receptors, internalized chemokines but did not effectively scavenge them. Instead, DARC mediated chemokine transcytosis, which led to apical retention of intact chemokines. Subsequently, a constant transcellular chemokine gradient led to more leukocyte migration across monolayers expressing DARC ${ }^{[59]}$. Despite the fact that the endothelial DARC activity fits perfectly in the inflammatory model underlying plaque formation there is no direct evidence linking DARC mediated pathways and atherosclerosis. In fact the only available data in the field of cardiovascular disease refer to the erythrocyte DARC and its ability to bind CXCL8. de Winter et al investigated whether rapid clearance of CXCL8 from plasma through binding to the erythrocyte DARC may be responsible for failure to detect CXCL8 consistently after acute myocardial infarction. The investigators measured plasma concentrations of CXCL8 and erythrocyte bound CXCL8 post myocardial infarction and concluded that CXCL8 was released in plasma after acute myocardial infarction and subsequently bound to red blood cells; resulting in only a transient rise of plasma CXCL8 and a more prolonged increase of erythrocyte bound CXCL8 ${ }^{[60]}$.

Erythrocytes have been also implicated in atherosclerotic plaque destabilization. Histopathology studies support this hypothesis since they have identified erythrocyte membranes in the atherosclerotic plaque and also have shown an association between the presence of erythrocytes and plaque vulnerability ${ }^{[12,13,61]}$. Haemorrhages within the plaque (intraplaque haemorrhage), arising from rupture of intraplaque micro-vessels and "healed" erosions of the atherosclerotic plaque have been proposed as major mechanisms by which erythrocytes can reach the atherosclerotic plaque ${ }^{[17,20]}$. Since erythrocytes carry on their surface DARC-bound pro-inflammatory chemokines it is possible that they release their proinflammatory load -including CXCL8-, when extravasated in lesion area. The latter phenomenon could initiate an inflammatory cascade that would lead to plaque rupture. We have recently reported an association between CXCL8 concentrations on erythrocyte membranes and clinical instability in coronary artery disease that further supports the above hypothesis ${ }^{[10,62]}$. However, the exact biochemical pathways following the extravasation of erythrocytes in the atherosclerotic milieu, the kinetics and activity of their membrane chemokines and the associated ligands has not been studied in detail and deserves further investigation.

\section{Future perspectives}

\section{Erythrocytes as regulators of inflammation in atherosclerosis}

Published data have shown that erythrocyte membranes are characterized by a multispecific cell surface receptor that has the capacity to bind a wide variety of inflammatory peptides of both major chemokine families ${ }^{[21,22]}$. The main characteristics of the DARC receptor may be summarized as follows: a) it is multi-specific; b) it is unable to initiate intracellular signals, and c) chemokines bound to erythrocyte surface are inaccessible to their normal target inflammatory cells ${ }^{[21,22]}$. DARC presence on erythrocytes' membranes and its unique functional features suggest a crucial role for these cells in the regulation of inflammatory processes.

In general cytokines are presumed to be circulating soluble plasma proteins ${ }^{[22]}$. No other cell type -apart from erythrocytes- is known to act as a reservoir for signal transduction peptides $^{[22]}$. Furthermore, the 1000-fold greater abundance of erythrocytes over immune-related circulating cells in blood accounts for the significance of their role in chemokine homeostasis in sites of vascular inflammation ${ }^{[22]}$.

More importantly, atherosclerotic vasa vasorum are characterized by compromised structural integrity ${ }^{[63]}$. The aforementioned leaky and fragile morphology of plaque neovessels may lead to extensive erythrocyte infiltration or to intraplaque haemorrhage ${ }^{[63]}$. Erythrocytes and their membranes' inflammatory arsenal could thus reach the atherosclerotic plaque and exert their regulatory role at local lesional level. A wide variety of inflammation-related modulator roles may be attributed 
to erythrocytes. Although not proven till today, erythrocytes can possibly release their chemokine load within the plaque thus fuelling the inflammatory process. Conversely, erythrocytes might scavenge locally produced chemokines with their uncoupled receptors therefore cooling the inflammatory cascade.

\section{From bench to bedside}

The role of erythrocytes as a sink for chemokines could inspire numerous diagnostic and therapeutic applications. The chemokine content of circulating erythrocyte membranes could be used as a prognostic marker of future acute ischemic events, since the augmentation of atherosclerotic lesion inflammation is well associated with plaque's instability. The density of uncoupled DARC receptors on erythrocyte membranes could on the other hand be used as a protective marker regarding clinical instability in coronary artery disease. Finally, the use of DARC receptors as vehicles for local delivery of inflammatory regulators -ie, CXC receptors blocking peptides reflects another therapeutic potential that deserves further investigation.

In conclusion, the key points of the present article can be summarised as follows:

Erythrocytes carry multispecific receptors on their membrane that can bind a wide array of chemokines. CXC chemokines -high affinity ligands for DARC receptors- have been shown to modulate the inflammatory cascade within the atherosclerotic plaque.

Erythrocytes have been identified within atherosclerotic plaques and their role in promoting plaque growth and instability has also been established.

Considerable more data will be needed to elucidate the role of erythrocyte DARC in atherosclerosis and establish its role as a novel diagnostic and therapeutic target.

\section{References}

1 Cutbush M, Mollison PL, The Duffy blood group system. Heredity 1950; 4: 383-9.

2 Lewis M, Kaita H, Chown B. The Duffy blood group in caucasians: A further population sample. Vox Sang 1972; 23: 523-7.

3 Pogo AO, Chaudhuri A. The Duffy protein: a malarial and chemokine receptor. Semin Hematol 2000; 37: 122-9.

4 Reich D, Nalls MA, Kao WH, Akylbekova EL, Tandon A, Patterson N, et al. Reduced neutrophil count in people of African descent is due to a regulatory variant in the Duffy antigen receptor for chemokines gene. PLoS Genet 2009; 5: e1000360.

5 Pruenster M, Rot A. Throwing light on DARC. Biochem Soc Trans 2006; 34: 1005-8.

6 Meny GM. The Duffy blood group system: a review. Immunohematology 2010; 26: 51-6.

7 Hadley TJ, Peiper SC. From malaria to chemokine receptor: the emerging physiologic role of the Duffy blood group antigen. Blood 1997; 89: 3077-91.

8 Neote K, Malk JY, Kolakowski LF, Schall TJ. Functional and biochemical analysis of the cloned Duffy antigen: identity with the red blood cell chemokine receptor. Blood 1994; 84: 44-52.

9 Hellings WE, Peeters W, Moll FL, Pasterkamp G. From vulnerable plaque to vulnerable patient: the search for biomarkers of plaque destabilization. Trends Cardiovasc Med 2007; 17: 162-71.

10 Tziakas DN, Chalikias GK, Stakos D, Boudoulas $\mathrm{H}$. The role of red blood cells in the progression and instability of atherosclerotic plaque. Int J Cardiol 2010; 142: 2-7.

11 Virmani R, Roberts WC. Extravasated erythrocytes, iron, and fibrin in atherosclerotic plaques of coronary arteries in fatal coronary heart disease and their relation to luminal thrombus: frequency and significance in 57 necropsy patients and in 2958 five mm segments of 224 major epicardial coronary arteries. Am Heart J 1983; 105: 788-97.

12 Arbustini E, Morbini P, D'Armini AM, Repetto A, Minzioni G, Piovella F, et al. Plaque composition in plexogenic and thromboembolic pulmonary hypertension: the critical role of the thrombotic material in pultaceous core formation. Heart 2002; 88: 177-82.

13 Kolodgie FD, Gold HK, Burke AP, Fowler DR, Kruth HS, Weber DK, et al. Intraplaque hemorrhage and progression of coronary atheroma. N Engl J Med 2003; 349: 2316-25.

14 Minetti M, Agati L, Malorni W. An emerging concept in cardiovascular disease is the role of the erythrocyte as a "barometer" of overall cardiovascular health. The microenvironment can shift erythrocytes from a friendly to harmful behavior: pathogenic implications for vascular diseases. Cardiovasc Res 2007; 75: 21-8.

15 Yeagle PL. Cholesterol and the cell membrane. Biochim Biophys Acta 1985; 822: 267-87.

16 Yuan XM, Brunk UT, Olsson AG. Effects of iron- and hemoglobin-loaded human monocyte-derived macrophages on oxidation and uptake of LDL. Arterioscler Thromb Vasc Biol 1995; 15: 1345-51.

17 Virmani R, Kolodgie FD, Burke AP, Finn AV, Gold HK, Tulenko TN, et al. Atherosclerotic plaque progression and vulnerability to rupture: Angiogenesis as a source of intraplaque hemorrhage. Arterioscler Thromb Vasc Biol 2005; 25: 2054-61.

18 Tziakas DN, Kaski JC, Chalikias GK, Romero C, Fredericks S, Tentes IK, et al. Total cholesterol content of erythrocyte membranes is increased in patients with acute coronary syndrome. J Am Coll Cardiol 2007; 49: 2081-9.

19 Tziakas DN, Chalikias GK, Stakos D, Tentes IK, Chatzikyriakou SV, Mitrousi $\mathrm{K}$, et al. Cholesterol composition of erythrocyte membranes and its association with clinical presentation of coronary artery disease. Coron Artery Dis 2008; 19: 583-90.

20 Moreno P, Purushothaman KR, Sirol M, Levy AP, Fuster V. Neovascularization in human atherosclerosis. Circulation 2006; 113 : 2245-52.

21 Neote K, Darbonne W, Ogez J, Horuk R, Schall TJ. Identification of a promiscuous inflammatory peptide receptor on the surface of red blood cells. J Biol Chem 1993; 268: 12247-9.

22 Darbonne WC, Rice GC, Mohler MA, Apple T, Hebert CA, Valente AJ, et al. Red blood cells are a sink for interleukin-8, a leukocyte chemotaxin. J Clin Invest 1991; 88: 1362-9.

23 Rubin O, Crettaz D, Tissot JD, Lion N. Microparticles in stored red blood cells: submicron clotting bombs? Blood Transfus 2010; 8: s31-s8.

24 Oreskovic RT, Dumaswala UJ, Greenwalt TJ. Expression of blood group antigens on red cell microvesicles. Transfusion 1992; 32: 848-9.

25 Xiong Z, Cavaretta J, Qu L, Stolz DB, Triulzi D, Lee JS. Red blood cell microparticles show altered inflammatory chemokine binding and release ligand upon interaction with platelets. Transfusion 2011; 51: 610-21.

26 Blum A. The possible role of red blood cell microvesicles in atherosclerosis. Eur J Intern Med 2009; 20: 101-5.

27 Luster AD. Chemokines - chemotactic cytokines that mediate inflammation. N Engl J Med 1998; 338: 436-45.

28 Apostolakis S, Papadakis EG, Krambovitis E, Spandidos DA. Chem- 
okines in vascular pathology (review). Int J Mol Med 2006; 17: 691701.

29 Shimizu K, Mitchell RN. The role of chemokines in transplant graft arterial disease. Arterioscler Thromb Vasc Biol 2008; 28; 1937-49.

30 Jin T, Xu X, Hereld D. Chemotaxis, chemokine receptors and human disease. Cytokine 2008; 44: 1-8.

31 Comerford I, Nibbs RJ. Post-translational control of chemokines: a role for decoy receptors? Immunol Lett 2005; 96: 163-74.

32 Zernecke A, Shagdarsuren E, Weber C. Chemokines in atherosclerosis: an update. Arterioscler Thromb Vasc Biol 2008; 28: 1897-908.

33 Apostolakis S, Vogiatzi K, Amanatidou V, Spandidos DA. Interleukin 8 and cardiovascular disease. Cardiovasc Res 2009; 84: 353-60.

34 Weber C, Schober A, Zernecke A. Chemokines: key regulators of mononuclear cell recruitment in atherosclerotic vascular disease. Arterioscler Thromb Vasc Biol 2004; 24: 1997-2008.

35 Barlic J, Murphy PM. Chemokine regulation of atherosclerosis. J Leukoc Biol 2007; 82: 226-36.

36 Weber KS, von Hundelshausen P, Clark-Lewis I, Weber PC, Weber C. Differential immobilization and hierarchical involvement of chemokines in monocyte arrest and transmigration on inflamed endothelium in shear flow. Eur J Immunol 1999; 29: 700-12.

37 Rus HG, Vlaicu R, Niculescu F. Interleukin-6 and interleukin-8 protein and gene expression in human arterial atherosclerotic wall. Atherosclerosis 1996; 127: 263-71.

38 Apostolopoulos J, Davenport P, Tipping PG. Interleukin-8 production by macrophages from atheromatous plaques. Arterioscler Thromb Vasc Biol 1996; 16: 1007-12.

39 Yue TL, Wang X, Sung CP, Olson B, McKenna PJ, Gu JL, et al. Interleukin-8. A mitogen and chemoattractant for vascular smooth muscle cells. Circ Res 1994; 75: 1-7.

40 Gerszten RE, Garcia-Zepeda EA, Lim YC, Yoshida M, Ding HA, Gimbrone MA Jr, et al. MCP-1 and IL-8 trigger firm adhesion of monocytes to vascular endothelium under flow conditions. Nature 1999; 398: 718-23.

41 Boisvert WA, Santiago R, Curtiss LK, Terkeltaub RA. A leukocyte homologue of the IL-8 receptor CXCR-2 mediates the accumulation of macrophages in atherosclerotic lesions of LDL receptor-deficient mice. J Clin Invest 1998; 101: 353-63.

42 Schwartz D, Andalibi A, Chaverri-Almada L, Berliner JA, Kirchgessner $\mathrm{T}$, Fang ZT, et al. Role of the GRO family of chemokines in monocyte adhesion to MM-LDL-stimulated endothelium. J Clin Invest 1994; 94 : 1968-73.

43 Huo Y, Weber C, Forlow SB, Sperandio M, Thatte J, Mack M, et al. The chemokine $\mathrm{KC}$, but not monocyte chemoattractant protein-1, triggers monocyte arrest on early atherosclerotic endothelium. J Clin Invest 2001; 108: 1307-14.

44 Mach F, Sauty A, larossi AS, Sukhova GK, Neote K, Libby P, et al. Differential expression of three $T$ lymphocyteactivating CXC chemokines by human atheroma-associated cells. J Clin Invest 1999; 104: 104150.

45 Gear AR, Camerini D. Platelet chemokines and chemokine receptors: linking hemostasis, inflammation, and host defense. Microcirculation 2003; 10: 335-46.

46 Abi-Younes S, Sauty A, Mach F, Sukhova GK, Libby P, Luster AD. The stromal cell-derived factor- 1 chemokine is a potent platelet agonist highly expressed in atherosclerotic plaques. Circ Res 2000; 86: 1318.

47 Schober A, Knarren S, Lietz M, Lin EA, Weber C. Crucial role of stromal cell-derived factor-1alpha in neointima formation after vascular injury in apolipoprotein E-deficient mice. Circulation 2003; 108 : 2491-7.
48 Petersen F, Bock L, Flad HD, Brandt E. Platelet factor 4-induced neutrophil-endothelial cell interaction: involvement of mechanisms and functional consequences different from those elicited by interleukin-8. Blood 1999; 94: 4020-8.

49 Engstad CS, Lia K, Rekdal O, Olsen JO, Osterud B. A novel biological effect of platelet factor 4 (PF4): enhancement of LPS induced tissue factor activity in monocytes. J Leukoc Biol 1995; 58: 575-81.

50 Pitsilos S, Hunt J, Mohler ER, Prabhakar AM, Poncz M, Dawicki J, et al. Platelet factor 4 localization in carotid atherosclerotic plaques: correlation with clinical parameters. Thromb Haemost 2003; 90: 1112-20.

51 Yu G, Rux AH, Ma P, Bdeir K, Sachais BS. Endothelial expression of Eselectin is induced by the plateletspecific chemokine platelet factor 4 through LRP in an NF-kappaB dependent manner. Blood 2005; 105 : 3545-51.

52 Nassar T, Sachais BS, Akkawi S, Kowalska MA, Bdeir K, Leitersdorf E, et al. Platelet factor 4 enhances the binding of oxidized low-density lipoprotein to vascular wall cells. J Biol Chem 2003; 278: 6187-93.

53 Rot A. Contribution of Duffy antigen to chemokine function. Cytokine Growth Factor Rev 2005; 16: 687-94.

54 Dawson TC, Lentsch AB, Wang Z, Cowhig JE, Rot A, Maeda N, et al. Exaggerated response to endotoxin in mice lacking the Duffy antigen/ receptor for chemokines (DARC). Blood 2000; 96: 1681-4.

55 Nebor D, Durpes MC, Mougenel D, Mukisi-Mukaza M, Elion J, HardyDessources MD, et al. Association between Duffy antigen receptor for chemokines expression and levels of inflammation markers in sickle cell anemia patients. Clin Immunol 2010; 136: 116-22.

56 Schnabel RB, Baumert J, Barbalic M, Dupuis J, Ellinor PT, Durda P, et al. Duffy antigen receptor for chemokines (Darc) polymorphism regulates circulating concentrations of monocyte chemoattractant protein-1 and other inflammatory mediators. Blood 2010; 115: 5289-99.

57 Lee JS, Frevert CW, Wurfel MM, Peiper SC, Wong VA, Ballman KK, et al. Duffy antigen facilitates movement of chemokine across the endothelium in vitro and promotes neutrophil transmigration in vitro and in vivo. J Immunol 2003; 170; 5244-51.

58 Mayr FB, Spiel AO, Leitner JM, Firbas C, Schnee J, Hilbert J, et al. Influence of the Duffy antigen on pharmacokinetics and pharmacodynamics of recombinant monocyte chemoattractant protein (MCP-1, CCL-2) in vivo. Int J Immunopathol Pharmacol 2009; 22: 615-25.

59 Pruenster M, Mudde L, Bombosi P, Dimitrova S, Zsak M, Middleton J, et al. The Duffy antigen receptor for chemokines transports chemokines and supports their promigratory activity. Nat Immunol 2009; 10 : 101-8.

60 de Winter RJ, Manten A, de Jong YP, Adams R, van Deventer SJ, Lie $\mathrm{KI}$. Interleukin 8 released after acute myocardial infarction is mainly bound to erythrocytes. Heart 1997; 78: 598-602.

61 Lin HL, Xu XS, Lu HX, Zhang L, Li CJ, Tang MX, et al. Pathological mechanisms and dose dependency of erythrocyte-induced vulnerability of atherosclerotic plaques. J Mol Cell Cardiol 2007; 43: 272-80.

62 Tziakas DN, Chalikias GK, Tentes IK, Stakos D, Chatzikyriakou SV, Mitrousi K, et al. Interleukin-8 is increased in the membrane of circulating erythrocytes in patients with acute coronary syndrome. Eur Heart J 2008; 29: 2713-22.

63 Sluimer JC, Kolodgie FD, Bijnens AP, Maxfield K, Pacheco E, Kutys B, et al. Thin-walled microvessels in human coronary atherosclerotic plaques show incomplete endothelial junctions relevance of compromised structural integrity for intraplaque microvascular leakage. J Am Coll Cardiol 2009; 53: 1517-27. 